\title{
Photoluminescence Properties of Nanocrystals
}

\author{
L. S. Cavalcante, ${ }^{1,2}$ J. C. Sczancoski, ${ }^{2}$ J. A. Varela, ${ }^{3}$ E. Longo, ${ }^{3}$ \\ J. Andrés, ${ }^{3}$ and S. K. Rout ${ }^{4}$ \\ ${ }^{1}$ Departamento de Química, Universidade Federal de São Carlos, P.O. Box 676, 13565-905 São Carlos, SP, Brazil \\ ${ }^{2}$ LIEC-IQ, Universidade Estadual Paulista, P.O. Box 355, 14801-907 Araraquara, SP, Brazil \\ ${ }^{3}$ Departamento de Química Física y Analítica, Universitat Jaume I, 12071 Castello, Spain \\ ${ }^{4}$ Department of Applied Physics, Birla Institute of Technology, Mesra, Ranchi 835215, India \\ Correspondence should be addressed to L. S. Cavalcante, laeciosc@bol.com.br
}

Received 11 December 2012; Accepted 11 December 2012

Copyright (C) 2012 L. S. Cavalcante et al. This is an open access article distributed under the Creative Commons Attribution License, which permits unrestricted use, distribution, and reproduction in any medium, provided the original work is properly cited.

Photoluminescence (PL) is an optical phenomenon exhibited by some semiconductor materials when excited by an electromagnetic radiation source. After the excitation and recombination processes, these kinds of materials are able to emit photons with less energy than those employed during the electronic excitation. Initially, the researches on the PL properties focused on crystalline materials under cryogenic conditions. However, from the technological viewpoint, the discovery of new materials with visible PL emissions at room temperature has become more interesting for the improvement or development of electrooptic devices. Currently, functional and practical applications of this physical phenomenon in our daily life can be found in light-emitting diodes, lasers, lamps, sensors, scintillators, electronic displays, and so on. In this special issue, the reader will find 10 selected papers briefly summarized below.

PL properties of silicon ( $\mathrm{Si}$ ) nanocrystals (NCs) were reported by $\mathrm{S}$. Kim et al. These authors studied the continuous-wave/time-resolved PL, cathodoluminescence, and electroluminescence of silicon Si NCs produced by ion beam sputtering deposition of $\mathrm{SiO} x$ single layer or $\mathrm{SiO} x / \mathrm{SiO}_{2}$ multilayers and subsequent annealing.

L. F. da Silva et al. investigated the difference of the PL properties in $\mathrm{SrTiO}_{3}$ micro- and nanocrystals prepared by microwave-assisted hydrothermal method, using two different titanium precursors. In addition, the $\mathrm{SrTiO}_{3}$ nanocrystals were characterized by means of X-ray diffraction, field emission scanning electron microscopy, high-resolution transmission electron microscopy, and X-ray absorption near edge structure spectroscopy.
The concentration effect of polyvinylpyrrolidone (PVP) as polymer surfactant on the PL properties of pure and Ni-doped zinc sulfide ( $\mathrm{ZnS}$ ) nanocrystalline thin films as explained by T. M. Thi et al. These authors investigated the PL excitation, time-resolved PL spectra, and PL decay time of these thin films, which were synthesized by the wet chemical method and spincoating.

H. W. Park and D.-H. Kim verified the effect of different solvents (water, hexane, and toluene) on the surface passivation of CdSe NCs in order to improve its visible luminescence. The results found in this research indicated that the water molecules promote an enhanced ligand passivation on the CdSe NCs and lead to a high PL emission with quantum yield $(\approx 85 \%)$ of these core CdSe quantum dots.

The PL properties of doped-N,S and $\mathrm{Mg}$ and pure $\mathrm{ZnO}$ nanocrystalline thin films prepared by the radio frequency magnetron sputtering method were reported by $\mathrm{H}$. Che et al. In this work, these authors studied several factors (structural defects, dopants, and intermediary levels) that act on the optical properties of transparent $\mathrm{ZnO}$ thin films. Moreover, there was a relation of PL properties with the presence of deep excitonic energy level in the direct band gaps of these films.

S. Wu et al. reported the effect of different ratio solvents (water/ethanol), use of tetraethylenepentamine as a new chelating ligand, rare earth $\left(\mathrm{Yb}^{3+} / \mathrm{Er}^{3+}\right)$ concentration and reaction time on the morphology and improvement of the PL properties of $\mathrm{NaYF}_{4}$ nanoparticles synthesized by the hydrothermal method. 
Two interesting papers on zinc oxide $(\mathrm{ZnO})$ nanostructures were presented by J. Z. Marinho et al. and S. L. Wang et al. In the first one, irregular $\mathrm{ZnO}$ microcrystals and spheres-like $\mathrm{ZnO}$ nanocrystals were prepared by the conventional water bath heating and microwave-assisted hydrothermal method, respectively. The effect of a different synthesis method using urea as partial reactant with the $\left(\mathrm{C}-\mathrm{NH}_{2}\right)$ groups promoted the formation of $\mathrm{ZnO}$ crystals with different structural defects. This factor acted directly on the PL behavior of $\mathrm{ZnO}$ micro- and nanocrystals. In the second one, the authors prepared propeller-like $\mathrm{ZnO}$ nanostructures by the chemical vapor deposition method and investigated its PL and photocatalytic properties. The results obtained in this work indicated that the propellerlike $\mathrm{ZnO}$ nanostructures have superior PL emission in the ultraviolet (UV) region when compared to the flowershaped $\mathrm{ZnO}$ nanostructures prepared by the same method. Moreover, after $3 \mathrm{~h}$ of UV-light illumination, the propellerlike nanostructures showed a higher photocatalytic activity for the degradation of rhodamine $\mathrm{B}$.

S.-Y. Ting et al. explained the improvement in the PL properties of $\mathrm{ZnO}$ nanocrystalline thin films, employing different buffer layers on sapphire substrates. In this work, these authors described that the effect of different GaN and $\mathrm{MgO}$ buffer layers promotes modifications in the local structure, formation of pores on the film surface, and appearance of deep levels at $550 \mathrm{~nm}$ in the PL emission spectra.

Finally, T. D. Hoanh et al. synthesized a new lightemissive material, called bis $(3 \mathrm{H}-1,2,3$-triazolo-[4,5-b]pyridine-3-ol)zinc $\left(\mathrm{Zn}(\mathrm{TAP})_{2}\right)$, by the chemical method. This new complex has excellent PL and electroluminescent properties, that is, interesting blue light emission and high luminance of $2,800 \mathrm{~cd} / \mathrm{m}^{2}$ at $12 \mathrm{~V}$. The obtained results in this work by the electrical measurements suggest that this complex can be a good candidate for the development of organic light emitting diodes.

As guest editors for this special issue, we are pleased with diverse and intense research activities in this area. We expect the special issue will be wellreceived by the reader as a small sample of the current research on the PL properties of nanocrystals and nanocrystalline thin films.

\section{Acknowledgments}

The guest editors would like to acknowledge and thank authors, reviewers, and staff members of Hindawi Publishing Corporation for their contributions and support to this special issue. 

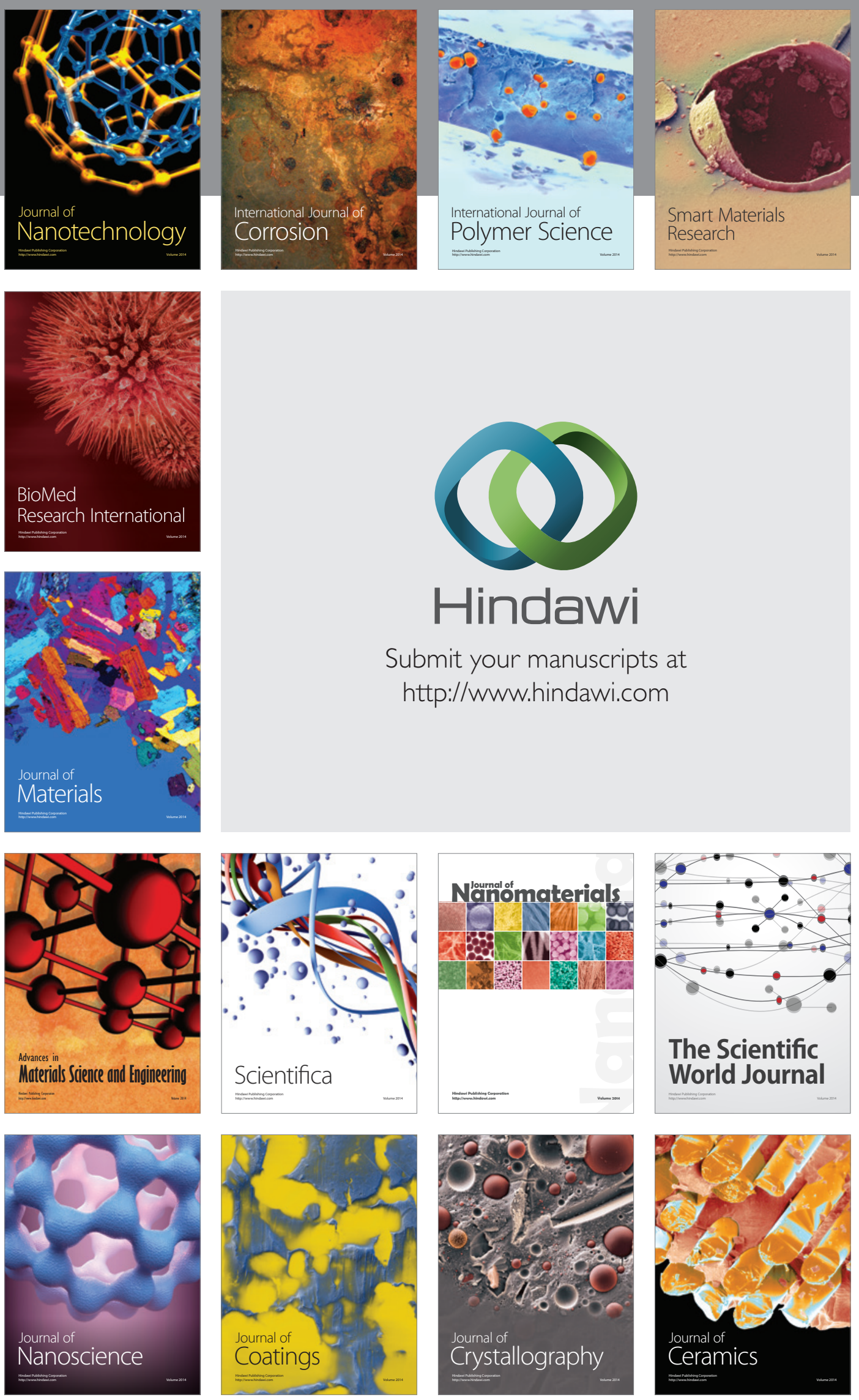

The Scientific World Journal

Submit your manuscripts at

http://www.hindawi.com

\section{World Journal}

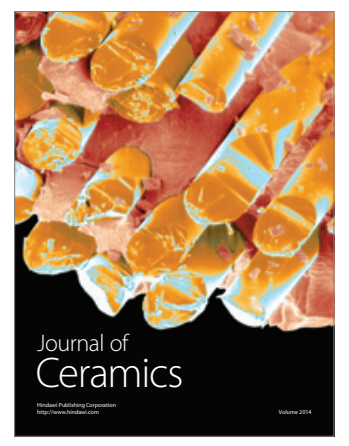

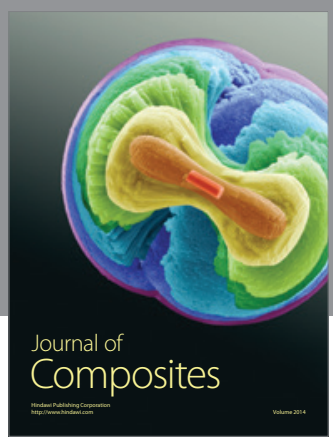
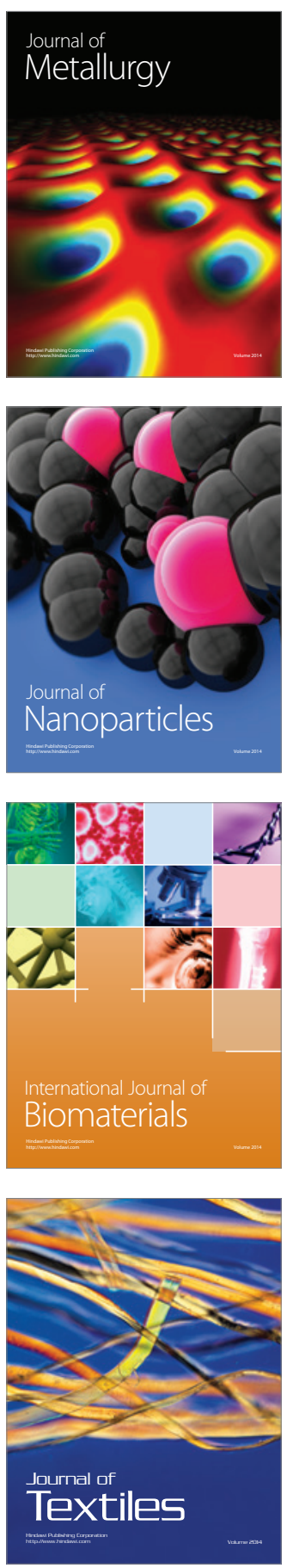